\title{
Misdiagnosed atypical paroxysmal kinesigenic dyskinesia: a case report
}

This article was published in the following Dove Press journal:

Neuropsychiatric Disease and Treatment

\author{
Fen $\operatorname{Pan}^{1,2}$ \\ Shangda $\mathrm{Li}^{3}$ \\ Haimei $\mathrm{Li}^{3}$ \\ Yi Xu ${ }^{1,2}$ \\ Manli Huang ${ }^{1,2}$ \\ 'Department of Psychiatry, The \\ First Affiliated Hospital, College \\ of Medicine, Zhejiang University, \\ Hangzhou 31 0003, China; ${ }^{2}$ The Key \\ Laboratory of Mental Disorder's \\ Management of Zhejiang Province, \\ Hangzhou 310003, China; ${ }^{3}$ School \\ of Medicine, Zhejiang University, \\ Hangzhou 310000, China
}

Background: Paroxysmal kinesigenic dyskinesia (PKD) is characterized by sudden episodes of involuntary movements. PKD is a very rare movement disorder, and correct clinical diagnosis is often a challenge.

Case: We present the case of a 23-year-old female with PKD. The patient showed episodes of twisting movements for 3 years. The symptoms lasted for about 5-10 minutes and subsided spontaneously. She was diagnosed as having epilepsy, and depressive and anxiety disorders successively. However, her symptoms did not alleviate after taking sodium valproate and antidepressants. Though there were no mutations in her PRRT2 gene, carbamazepine was used for treatment and was effective in controlling her symptoms.

Conclusion: The clinical features of PKD patients are not always typical; therefore, it is important to distinguish PKD from the other subtypes of paroxysmal dyskinesia and psychogenic disorders.

Keywords: paroxysmal kinesigenic dyskinesia, misdiagnosis, PRRT2 gene

\section{Introduction}

Paroxysmal kinesigenic dyskinesia (PKD) is characterized by recurrent attacks of involuntary movements, which are triggered by sudden voluntary movements. ${ }^{1}$ Attacks of PKD include combinations of dystonia, choreoathetosis, and ballism and do not involve loss of consciousness. They typically last less than 1 minute, and occur as often as 10-100 times per day. The age at onset is between 1 and 20 years if there is no family history (age at onset has more variable in those with family history). The gene responsible for PKD was recently identified as PRRT2. ${ }^{2}$ The attacks of PKD can be prevented or reduced in frequency by phenytoin or carbamazepine. ${ }^{3}$ To the best of our knowledge, there are very few reports of atypical attacks in patients with PKD. In this report, we present the case of a 23-year-old young female with atypical PKD attacks.

\section{Case}

A 23-year-old Chinese woman was admitted to our hospital because of a 3-year history of attacks of involuntary movements. Three years earlier, one day, the patient took 4 exams continuously and felt tired at the 4th exam. Suddenly, her bilateral limbs rolled outward and jerked convulsively. Her wrists and ankles bent transiently, and her muscles were stiff. She staggered and could not coordinate the movements of her arms and legs. There were no speech and swallowing changes noticed during the attacks. Her jaw and facial regions were also not affected. She was conscious during the episode with a little chest tightness and palpitation. The symptoms lasted for about 5-10 minutes and then spontaneously improved. Since then, the attacks have kept occurring 1-2 times 
per month and are mostly when she is tired. The patient also complained that she had a premonition before the attacks. She visited the neurological department of our institution and was diagnosed as having epilepsy. She complained that the symptoms occurred more frequently after being treated with sodium valproate for 1 month. The patient again visited the neurological department and was diagnosed as having major depressive disorder. However, there was no improvement in her symptoms after taking flupentixol.

Finally, the patient was admitted to our hospital with a diagnosis of anxiety disorder. The results of cranial computed tomography angiography and MRI enhanced scan were normal. There were no abnormities in her active electroencephalogram, electromyogram, echocardiography, electrocardiogram, myocardial enzymogram, blood routine test, thyroid function, liver panel examinations, renal function, and serum electrolytes. Her neurological signs were negative. A full psychiatric assessment revealed symptoms of anxiety. She scored 176, 41, and 59 points, respectively, in the Symptom Checklist-90 (SCL-90), Self-Rating Depression Scale (SDS), and Self-Rating Anxiety Scale (SAS). An SCL-90 score $\geq 160$ indicates a positive screening result. An SDS score $<53$ indicates the patient is normal. The SAS scores in the range from 50 to 60 indicate mild anxiety. The patient reported no history of trauma, any other somatic disease, or alcohol or substance use. She also reported no family history of psychiatric and neurological disease. She was prescribed paroxetine $(20 \mathrm{mg}$ per day), however her symptoms were not well controlled and occurred once during hospitalization. The most recent episode occurred when the patient was sleeping, which was triggered by a change in the sleeping position. While lying on the bed, her bilateral limbs rolled outwards involuntarily. She was awake and could do some voluntary movements like covering herself with the quilt. According to these symptoms and her medical history, we diagnosed the patient as having PKD, although her gene sequencing showed no mutation in kinesigenic dyskinesia relative gene PRRT2. Her medicine was replaced with carbamazepine (200 mg twice daily). The patient reported that she felt better after taking carbamazepine for a few days. She came back to our department for a follow-up visit after 2 months and reported that there was no attack of PKD after discharge. Unfortunately, the patient got serious drug eruptions and was covered in pruritic red macules and papules, except for her face. She had to stop the medicine, and the eruptions gradually improved within a couple of weeks. The symptoms reappeared twice after stopping carbamazepine. Oxcarbazepine was used to treat the patient 2 weeks later after the rash subsided. She reported that oxcarbazepine also relieved her symptoms, but was not as effective as carbamazepine. The attacks occurred once per month and lasted for about 2 minutes.

\section{Discussion}

PKD is an autosomal dominant disorder characterized by episodic and recurrent dystonic or choreoathetoid attacks triggered by initiation of voluntary movements. The onset of PKD is usually in childhood. The attacks can involve 1 or multiple limbs, typically last less than a minute, and occur up to 100 times per day. ${ }^{4}$ The disorder is eminently treatable with antiepileptic drugs, such as phenytoin and carbamazepine. $^{5}$

PKD must be differentiated from the other subtypes of paroxysmal dyskinesia, comprising paroxysmal nonkinesigenic dyskinesia (PNKD) and paroxysmal exerciseinduced dyskinesia (PED). Attacks of PNKD are usually triggered by alcohol, coffee, or strong emotion and last longer than attacks of PKD, often from 10 minutes to 1 hour. PNKD usually occurs only a few times a year, which is less frequent than PKD. ${ }^{6}$ In PED, episodes last from 5 minutes to an hour, occur once or twice per month, and are induced by physical exertion after long periods of exercise. ${ }^{7}$ The age at onset of our patient was 21 years old. Her symptoms lasted for about 5-10 minutes during every episode and occurred 1-2 times per month. However, her symptoms were not typical enough to be diagnosed as PKD. Also, the attacks were not triggered by alcohol, coffee, strong emotion, or long periods of exercise. Moreover, carbamazepine was obviously effective for our patient, while PNKD and most cases of PED do not respond to antiepileptic drugs. Atypical PKDs hardly ever been reported, although the frequency of attacks in 4 children ranged from 1 to 10 per month (typically occurring as often as 10-100 times per day) according to a previous report. ${ }^{8}$ The age at onset and symptom features of the patient in the present case were not typical, which increased the difficulty of diagnosis. Further studies are needed to systematically explore the atypical subtypes of PKD for accurate diagnosis and appropriate treatments.

PKD can also be misdiagnosed as epilepsy and anxiety disorder. ${ }^{9,10}$ The patient in the present case was also misdiagnosed as having epilepsy, as she presented with attacks of involuntary movements. However, no spike wave was found in her 3 active electroencephalograms, and she did not respond to sodium valproate. The neurologists ruled out the PKD diagnosis based on her gene detection result. The gene responsible for PKD was recently identified as PRRT2. Mutations in the PRRT2 gene have been found in $61.5 \%-100 \%$ of patients with familial PKD and $12.5 \%-50 \%$ of patients with sporadic PKD. ${ }^{2}$ The patient in the present 
case reported no family history of PKD, and her gene sequencing showed no mutation in PRRT2. She visited our department and complained of feeling a little chest tightness and palpitation during attacks. She was worried about her health and feared that the symptoms would reappear. Her SAS score was 59, which indicated mild anxiety. She was admitted to our hospital with a diagnosis of anxiety disorder. However, the poor treatment effects made us rethink the diagnosis and treatment. Our case highlights the importance of the differential diagnosis of PKD from psychogenic diseases because the patients usually report fears and worries. Abhishek et al have reported the case of a 40-year-old man with PNKD who was initially diagnosed as having a psychogenic disorder. ${ }^{11}$

Moreover, paroxysmal dyskinesia is a rare movement disorder, and most physicians do not have a thorough understanding of it. The negative results of gene detection and symptoms of anxiety may lead to misdiagnosis.

As for the treatment, the patient responded dramatically to carbamazepine. She felt better after taking carbamazepine for a few days, and there was no attack of PKD in 2 months. However, serious drug eruptions occurred, and her medicine had to be changed to oxcarbazepine. There was 1 PKD attack in the first 2 weeks, and the patient did not feel as good as when taking carbamazepine. Then, our patient reported in the most recent follow-up visit that attacks had occurred once in the last month and lasted for about 2 minutes. Carbamazepine and oxcarbazepine are thought to be similarly effective and tolerable in the treatment of PKD. Although, some patients were found to have better tolerance when carbamazepine was replaced with oxcarbazepine for adverse symptoms of rash, drowsiness, diplopia, and nervousness, ${ }^{12}$ which was also validated in our case. In addition, it may be interesting to compare the onset of action between these 2 drugs.

\section{Conclusion}

The clinical features of PKD patients are not always typical; therefore, it is important to distinguish PKD from the other subtypes of paroxysmal dyskinesia and psychogenic disorders.

\section{Acknowledgments}

The authors thank the patient for her consent to publish this article. Written informed consent was obtained from the patient for the case details to be published. This work was supported by grant 2016YFC1307005 from the National Key Research and Development Program of China, grant LGF18H090003 of Basic Public Welfare Research Projects in Zhejiang Province, and grant 2015C03040 of Major Subject of Zhejiang Province. The funding sources had no role in the study design, collection, analysis, and interpretation of data, writing of the manuscript, or decision to submit the paper for publication.

\section{Disclosure}

The authors report no conflicts of interest in this work.

\section{References}

1. Demirkiran M, Jankovic J. Paroxysmal dyskinesias: clinical features and classification. Ann Neurol. 1995;38(4):571-579.

2. Chen WJ, Lin Y, Xiong ZQ, et al. Exome sequencing identifies truncating mutations in PRRT2 that cause paroxysmal kinesigenic dyskinesia. Nat Genet. 2011;43(12):1252-1255.

3. Spacey S, Adams P. Familial paroxysmal kinesigenic dyskinesia. In: Adam MP, Ardinger HH, Pagon RA, et al, editors. GeneReviews ${ }^{\circledR}$ Seattle, WA: University of Washington; 1993.

4. Bruno MK, Hallett M, Gwinn-Hardy K, et al. Clinical evaluation of idiopathic paroxysmal kinesigenic dyskinesia: new diagnostic criteria. Neurology. 2004;63(12):2280-2287.

5. Bhatia KP. Familial (idiopathic) paroxysmal dyskinesias: an update Semin Neurol. 2001;21(1):69-74.

6. Bhatia KP. The paroxysmal dyskinesias. J Neurol. 1999;246(3): 149-155.

7. Lance JW. Familial paroxysmal dystonic choreoathetosis and its differentiation from related syndromes. Ann Neurol. 1977;2(4):285-293.

8. Sun W, Li J, Zhu Y, Yan X, Wang W. Clinical features of paroxysmal kinesigenic dyskinesia: report of 24 cases. Epilepsy Behav. 2012;25(4) 695-699.

9. Mallik R, Nandi SS. Paroxysmal kinesigenic dyskinesia. J Assoc Phys Ind. 2016;64(4):77-78.

10. Kunii Y, Matsuda N, Yabe H. A case of paroxysmal kinesigenic dyskinesia which exhibited the phenotype of anxiety disorder. Neuropsychiatr Dis Treat. 2017;13:2181-2184.

11. Abhishek, Anand KS, Premsagar IC. Sporadic paroxysmal nonkinesigenic dyskinesia: a frequently-misdiagnosed movement disorder Singapore Med J. 2007;48(9):e250-e252.

12. Yang $\mathrm{Y}, \mathrm{Su} \mathrm{Y}$, Guo Y, et al. Oxcarbazepine versus carbamazepine in the treatment of paroxysmal kinesigenic dyskinesia. Int $J$ Neurosci. 2012;122(12):719-722.
Neuropsychiatric Disease and Treatment

\section{Publish your work in this journal}

Neuropsychiatric Disease and Treatment is an international, peerreviewed journal of clinical therapeutics and pharmacology focusing on concise rapid reporting of clinical or pre-clinical studies on a range of neuropsychiatric and neurological disorders. This journal is indexed on PubMed Central, the 'PsycINFO' database and CAS,

\section{Dovepress}

and is the official journal of The International Neuropsychiatric Association (INA). The manuscript management system is completely online and includes a very quick and fair peer-review system, which is all easy to use. Visit http://www.dovepress.com/testimonials.php to read real quotes from published authors. 